\title{
Visual, textual or hybrid: the effect of user expertise on different explanations
}

\author{
MAXWELL SZYMANSKI, Dept. of Computer Science, KU Leuven \\ MARTIJN MILLECAMP, Dept. of Computer Science, KU Leuven \\ KATRIEN VERBERT, Dept. of Computer Science, KU Leuven
}

As the use of AI algorithms keeps rising continuously, so does the need for their transparency and accountability. However, literature often adopts a one-size-fits-all approach for developing explanations when in practice, the type of explanations needed depends on the type of end-user. This research will look at user expertise as a variable to see how different levels of expertise influence the understanding of explanations. The first iteration consists of developing two common types of explanations (visual and textual explanations) that explain predictions made by a general class of predictive model learners. These explanations are then evaluated by users of different expertise backgrounds to compare the understanding and ease-of-use of each type of explanation with respect to the different expertise groups. Results show strong differences between experts and lay users when using visual and textual explanations, as well as lay users having a preference for visual explanations which they perform significantly worse with. To solve this problem, the second iteration of this research focuses on the shortcomings of the first two explanations and tries to minimize the difference in understanding between both expertise groups. This is done through the means of developing and testing a candidate solution in the form of hybrid explanations, which essentially combine both visual and textual explanations. This hybrid form of explanations shows a significant improvement in terms of correct understanding (for lay users in particular) when compared to visual explanations, whilst not compromising on ease-of-use at the same time.

CCS Concepts: • Human-centered computing $\rightarrow$ Empirical studies in visualization; User studies; User models; $\bullet$ Information systems $\rightarrow$ Decision support systems; • Computing methodologies $\rightarrow$ Artificial intelligence.

Additional Key Words and Phrases: XAI, visual explanations, textual explanations, domain expertise

\section{ACM Reference Format:}

Maxwell Szymanski, Martijn Millecamp, and Katrien Verbert. 2021. Visual, textual or hybrid: the effect of user expertise on different explanations. In Woodstock '18: ACM Symposium on Neural Gaze Detection, fune 03-05, 2018, Woodstock, NY. ACM, New York, NY, USA, 16 pages. https://doi.org/10.1145/1122445.1122456

\section{INTRODUCTION}

Many of the intelligent systems that we use daily, rely behind the scene on artificial intelligence (AI) which we do not always understand [28, 32]. However, as we increasingly depend on these systems to make decisions, there is also an increasing demand to make these systems more transparent, understandable and accountable [4]. Problematically, many of these techniques often appear as black-boxes by nature which means that it is difficult to get insight in their internal reasoning [1]. To address this issue, explainable AI (XAI) proposes algorithmic transparency by offering several techniques in generating explanations that focus on algorithmic decision-making [1].

Permission to make digital or hard copies of all or part of this work for personal or classroom use is granted without fee provided that copies are not made or distributed for profit or commercial advantage and that copies bear this notice and the full citation on the first page. Copyrights for components of this work owned by others than ACM must be honored. Abstracting with credit is permitted. To copy otherwise, or republish, to post on servers or to redistribute to lists, requires prior specific permission and/or a fee. Request permissions from permissions@acm.org.

(C) 2021 Association for Computing Machinery.

Manuscript submitted to ACM 
However, according to the work of Ribera et al. [29], the goal of XAI depends on the type of end user that is on the receiving end of the explanations. Developers and AI experts might for example use the explanations to verify that the system is working as expected, whereas lay users mostly use the explanations to understand their own situation [4]. One of the open challenges in the domain of XAI is the tailoring of explanations to the expertise of users [15]. In addition, little work has been done to evaluate whether the proposed methods indeed enhance human interpretability [34]. Previous research in the area of recommender systems has for instance shown that simpler justifications are often preferred over more complex explanations [16], but in the more general XAI field little work has been done so far to compare the utility of different explanations methods with actual end-users $[1,11]$.

To address these challenges, we compare in this study the utility of different types of explanations on top of an existing predictive algorithm with expert and lay users. To do so, we developed textual or visual explanations on top of an algorithm that predicted the reading time of a news article. A comparison was then made between users of different expertise groups to detect any potential differences in interpretation between both explanation types. Additionally, a new type of hybrid explanation was developed and evaluated which essentially combined both a visual and textual explanation. The research mainly focuses on the following questions:

RQ1: What impact do different types of explanations (textual, visual) have on the interpretation of a predictive algorithm?

The first question this research answers is in which way different representations of an explanation impact the understanding of the underlying information. This is done by prompting the users with several tasks that gauge their understanding of the explanation at hand, and seeing whether or not they are able to successfully find the information contained within the explanation.

RQ2: In which way are the differences in interpretation influenced by user expertise?

The second question this research answers is whether the expertise of the users moderates the interpretations of the explanations. This is done by recruiting users with different expertise (lay users and AI experts).

RQ3: What might potentially minimize these differences in interpretation?

The last question this study answers is whether we can improve the understandability of explanations by redesigning the explanations. This is done by implementing and evaluating a novel design of explanations which addresses the issues we discovered with the visual and textual explanations.

\section{RELATED WORK}

\subsection{Explaining Artificial Intelligence}

Due to the explosive growth in the use of AI applications that impact our daily life, people are increasingly aware of the black-box nature of these applications $[2,8]$. As a consequence, there is an increasing demand to make these applications more intelligible to humans by providing explanations, so called explainable AI (XAI) [15]. Moreover, due to the GDPR legislation there is also legal pressure to invent new AI techniques that are capable of making decisions more transparent to the end users $[1,3]$.

However, providing transparency is not the only benefit of explanations. Tintarev et al. [33] listed seven different purposes an explanation can serve: next to transparency, explanations could aim to make the system scrutable, to increase the trust of users in the system, to make the system more effective, to persuade users to consume an item, to help users to be more efficient and to increase the satisfaction of the users. Unfortunately, it is nearly impossible to create 
explanations that do well on all seven criteria which is the reason we focused mainly on transparency, effectiveness and efficiency [33].

\subsection{Designing good explanations}

Recently, several researchers in the field of XAI have argued that most of the guidelines for designing explanations are based purely on intuition or experience and lack theoretical knowledge of human decision making, psychology, philosophy or cognitive science $[1,8,24,36]$. As a consequence, these guidelines provide little justification on why they work or not [36]. In this study, we want to overcome this limitation by building upon the work of Hohman et al. [17] which offers an overview of capabilities that explanations can support. This list was synthesized based on the results of both a literature survey and a formative study through a series of interviews. The capabilities an explanation can support are:

- C-Local: Local instance explanation - quantifying each parameter's contribution to an instance prediction.

- C-CompaRIson: Instance explanation comparison - given a collection of instances, what parameters lead to their predictions.

- C-Counter: Counterfactuals - given an instance, ask what-if questions to observe the effect of changing a parameter on the prediction.

- C-NN: Nearest neighbor - find instances that have similar predictions or parameters to a given instance.

- C-ERRor: Regions of error - locate regions where model predictions are uncertain.

- C-ImportancE: Feature importance - give the parameters that are most influential to the overall predictions.

\subsection{Classification of explanations}

Due to the broad range of machine learning techniques and the growing body of research in XAI, there exist a wide variety of explanation techniques which makes it difficult to choose an appropriate design $[1,25]$. To assist AI developers in choosing the right technique, several researchers already proposed taxonomies to provide a holistic view of the XAI field. For example, the taxonomy of Arrieta et al. [3] classifies explanation techniques in a tree, with as first two layers (i) post-hoc explanations or transparent models and (ii) model-agnostic or model-specific explanation techniques. Another study proposes to identify research in the XAI field based on four different axes: XAI method, XAI measurement, XAI perception and XAI antithesis [1]. However, we prefer the taxonomy of Mohseni et al. [25] which uses five high-level characterizations to classify XAI explanations.

The first characterization they propose is the scale of the explanation which can be global or local. A global explanation explains the working of the overall machine learning model while a local explanation aims to only explain the relationship between specific input and the resulting output [25].

Similar to the taxonomy of Arrieta et al. [3], they also propose to classify explanation techniques based on whether post-hoc methods explain black-box machine learning algorithm or whether interpretable machine learning models are used.

A third characterization Mohseni et al. [25] propose, is the question to which the explanation provides an answer. It could for example provide an answer to the question why some output is produced, but also why not something else is produced, so called counterfactual explanations. An example of a system with such counterfactual explanations is ViCE which provides visual counterfactual explanations to explain why the system did not produce another output [13]. Other questions an explanation could answer are what-if, how-to and what-else questions. 
A fourth characterization that can be used to classify explanations is the way explanations are presented [25]. Explanations could be visual in the form of a graph such as a saliency map [1], but they can also be textual in the form of words and phrases or they can be analytical which allows users to explore the data and the model. Despite the large interest each of these methods received individually, there are only a few studies that compare these different presentation methods to learn when, why and for who they work [19, 27]. A noticeable exception that investigated the effect of different explanation presentations is the study of Kouki et al. [21] which found that textual explanations are more persuasive than visual explanations in a recommender system.

The fifth and last characterization is the type of users using the explanation. Similar to Ribera et al. [29], Mohseni et al. [25] categorized users in three different groups based on their expertise: (i) developers and AI experts who have a notion of what an AI system entails, (ii) domain experts who have knowledge on the area on which the AI system operates and (iii) lay users who do not have any AI knowledge nor domain expertise. Especially for this characterization, several researchers already argued that the users are a key aspect to be considered when designing an explanation $[3,8,31]$. Moreover, Gunning et al. [15] identified that one of the open challenges in XAI is tailoring the explanations to the expertise of end user. To address this challenge, this study will investigate how users with a different expertise interact and perceive different explanations.

\section{FIRST ITERATION: COMPARING TEXTUAL AND VISUAL EXPLANATIONS}

The main focus of this research is to offer local, instance-level explanations to the end user based on an underlying predictive model. The method for providing explanations is developed to work with any predictive model that meets the following criteria:

- INPUT: $n$ input variables $\left(v_{1}, \ldots, v_{n}\right)$, each spanning over a finite range of values, either continuous, discrete or ordinal.

- OutPut: 1-dimensional output variable $y$, with a range also either continuous, discrete or ordinal.

The output of the prediction can be any arbitrary function in terms of the main or quadratic effects (e.g. the explanations are not designed to handle interaction effects $v_{i} v_{j}$ where $i \neq j$ ) [14].

In our work, we evaluate explanations of predictive models in the context of predicting the average reading time of a magazine article, given the following input parameters: word count, topic relevance, article size, pictures used, and what page the article is on. Both visual and textual explanation designs were implemented in this first iteration which will be explained in detail in the next paragraphs.

\subsection{Visual explanation design}

To explain the prediction of the reading time in a visual way, we implemented a partial dependence plot (PDP) which is a well-documented approach for offering visual explanations [22]. A PDP is a two-dimensional plot that shows how the output $y$ (on the y-axis) is influenced by an individual input parameter $v_{i}$ (on the $\mathrm{x}$-axis). Figure 1 shows an example of a PDP used in this study. The reading time is plotted on the y-axis and the input parameter is here the number of words of the article (word count). On this figure, it is also shown that this design supports multiple capabilities identified by Hohman et al. [17], discussed in Section 2. Label 1 on Figure 1 points to the current value which denotes the C-LocAL capability that indicates how the selected parameter influences the prediction. Label 2 on this figure points to the graph itself which denotes the C-CounTER capability since it indicates how the prediction would change if the selected input is modified. 


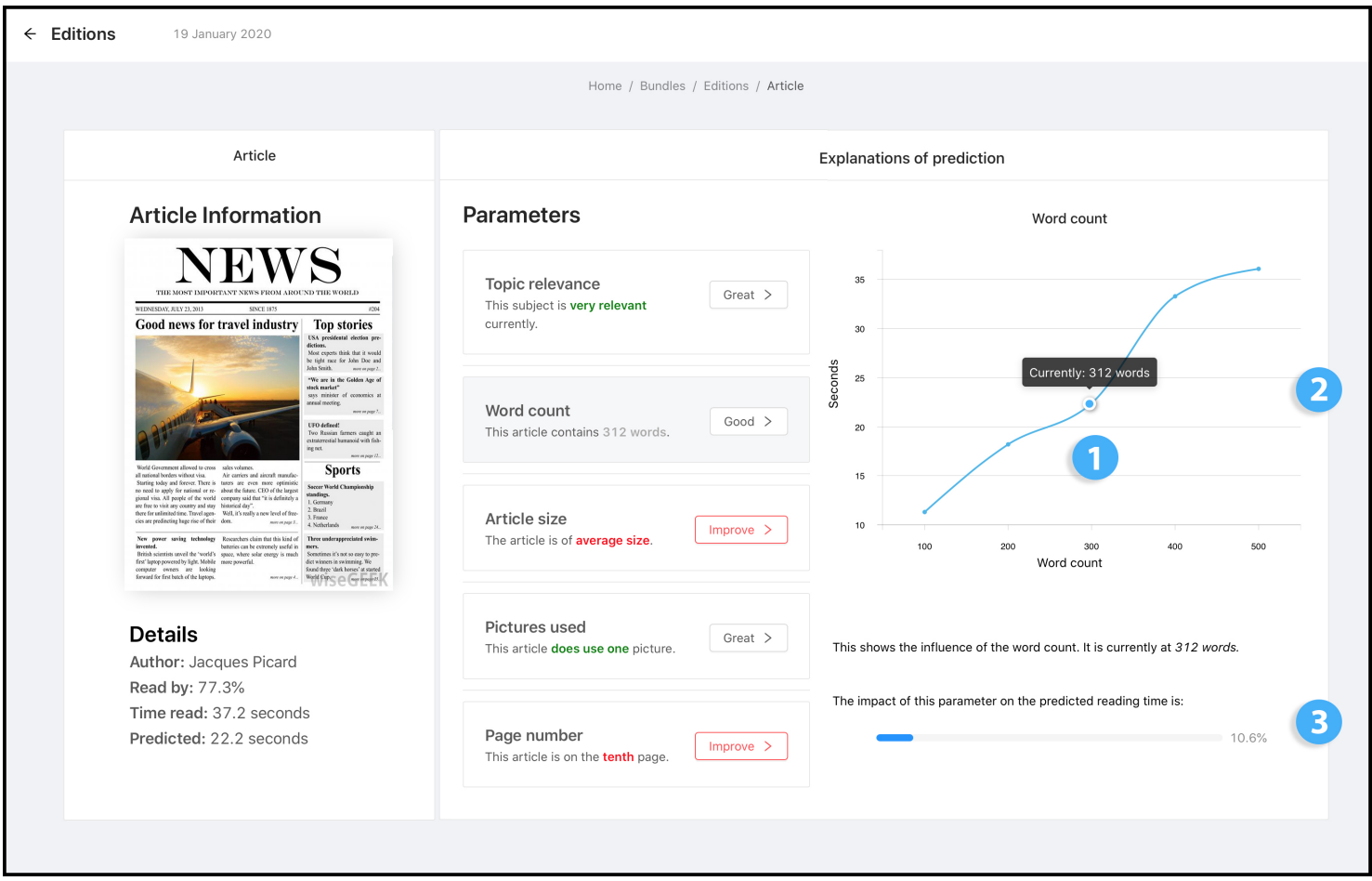

Fig. 1. Visual explanations for a selected instance. The current value (1) denotes the C-LocAL capability, (2) denotes C-Counter by indicating how the output would differ by changing the input and (3) denotes C-IMPORTANCE by showing the weight of the selected parameter.

Additionally, the PDP is accompanied by a bar underneath that indicates the weight of an individual parameter $v_{i}$ on the prediction. This value will be high if the change of the parameter has a relatively strong effect on the output. This bar supports the C-IMPORTANCE capability and is shown on Figure 1, label 3.

\subsection{Textual explanation design}

To explain the prediction of the reading time in a textual form, we generated a sentence per input parameter $v_{i}$ using the template described in [18]:

"The current value of the parameter $v_{i}$ is [...] (1) and increasing its value would have a [high/medium/low] (2) [positive/negative] (3) impact on the prediction."

To enable a fair comparison, these textual explanations contained the same information as the visual explanations.

Here, (1) denotes the explanation capability C-Local, (2) denotes the weight that the respective parameter has on the prediction (C-IMPORTANCE) and (3) explains whether increasing or decreasing the parameter has a positive impact on the predicted output (C-COUNTER). The interface with textual explanations is presented in Fig. 2. 


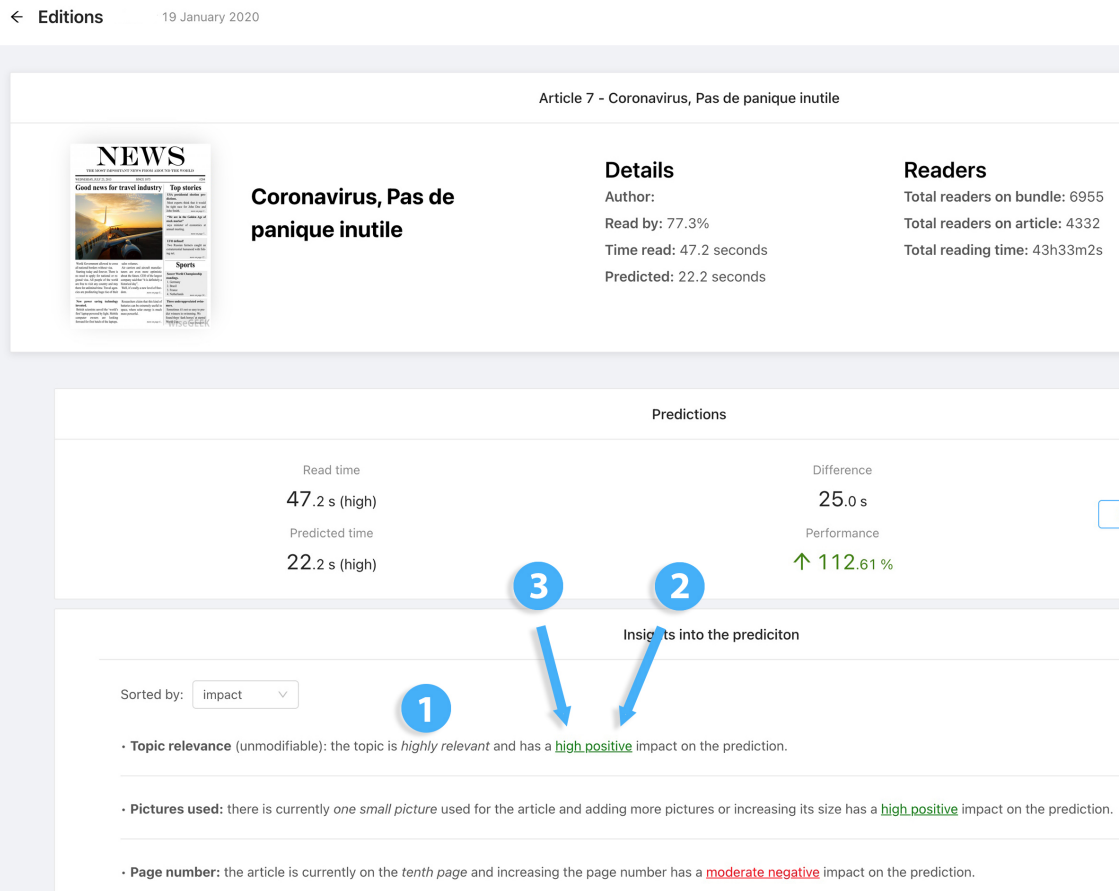

Fig. 2. Textual explanations for a selected instance. The current value (1) denotes the C-LocAL capability, how the selected parameter influences the prediction (2) denotes C-Counter and (3) denotes C-IMPortance.

\subsection{First User Study Methodology}

A first user study is designed to compare the differences in interpretation between visual and textual explanations (RQ1), and whether this interpretation is moderated by expertise (RQ2). We conducted a within-subject user study with 20 participants: 10 expert users (software engineers and AI developers, age between 25 and 53, 8M:2F) and 10 lay users (without any software or domain knowledge, age between 23 and 55, 3M:7F). Users were first instructed to explore the interface to get acquainted with the explanations after which they were given two tasks to fulfill:

- Task 1: Give two parameters with the highest impact on the prediction output.

- Task 2: What input parameter would you change and how, to maximally increase the predicted output.

Both tasks were selected from Hohman et al. [17] to test a capability, with the first task focusing on C-IMPORTANCE and the second task on C-CounTER. To address the issue that most XAI research is not tested with realistic tasks, both of these tasks are a realistic use case which was verified by a domain expert [7].

After fulfilling the tasks, the users were prompted with subjective-scale post-task questions about ease of use and time needed to perform the tasks using a 5-point Likert scale (with Q-AMOUNT and Q-CoMPLEx being phrase completion Likert scale questions) [9, 25]. These questions are:

- Q-InTuitive: "The explanations were intuitive to use."

- Q-EASEOFUsE: "The explanations were easy to interpret and understand."

- Q-Time: "I needed a lot of time to interpret the explanations." 
- Q-CoMpLEx: "The explanations were rather complex (high)/simple (low)."

- Q-Aмоunт: "The information given by the explanation was too much (high)/too little (low)."

Users had to perform the same tasks, and answer the same question for both the visual, as well as the textual explanations (albeit on different articles with different possible answers). The order of assignment was decided using a Latin square to ensure an orthogonal experimental design.

Additionally, we used the Think Aloud protocol (TA) to capture thoughts of participants when interacting with the explanations. This approach allowed users to give additional comments about their interpretation of explanations and gives us insight into the users reasoning.

After using both types of explanations, the users were prompted two open questions to gain insight into their use of those explanations:

- Q1: Which type of explanation do you prefer and why?

- Q2: In what use case would you use the explanations?

\subsection{First User Study Results}

To detect differences in responses to the aforementioned questions, we used a Wilcoxon signed-rank test since it does not make an assumption about the underlying distribution of the data, and takes the ordinal and paired nature of the questions into account [26,37]. Considering the fact that the user groups are relatively small (10 users per group), and that the Wilcoxon signed rank test is non-parametric, a significance level of $\alpha=0.1$ is used to detect differences between populations [20].

The results of this analysis revealed only one significant result $(U=6, p=.0745)$, i.e. expert users perceive that there is more information in the visual explanation $(2.63 \pm 0.40)$ than in the textual explanations $(2.25 \pm 0.53)$.

Additional to these post-task questions, we also captured the number of correctly answered questions. Figure $3 \mathrm{a}$ depicts the percentage of correctly answered tasks (both task 1 and task $\mathbf{2}$ combined). In general, users perform better with textual explanations than with visual explanations (with an average of $65 \%$ compared to $40 \%$ correct answers). If we split the results between experts and lay users, we noticed that for both the text and the visual explanations lay users perform worse than the experts. Especially for the visual explanations, the performance of lay users is really low with only $5 \%$ of correctly answered tasks.

As discussed in the previous section (Section 3.3), participants were asked to answer two open questions at the end of the study. For the first question, users needed to indicate which of the two explanations they preferred and why. The results indicated that $80 \%$ of users preferred the visual explanation. Similar to the previous analysis, we again divided participants based on experience, which revealed that experts have a stronger preference for visual explanations than lay users, as shown on Figure 3b. To analyze why users preferred visual or textual explanations, we identified different themes in their answers based on the key information they contain. We used the same approach to analyze the second open question about the context in which they would use the explanation. The themes that we identified in both questions can be found in Table 1 and will be discussed in the next paragraph.

\section{Q1: WHICH EXPLANATION DO YOU PREFER AND WHY?}

As shown in Table 1 there are four experts who prefer visual explanations due to their academic or professional expertise. For example, U1 responded: "I prefer the visual explanations because I work daily graphs". Three other experts mentioned that they prefer visual explanations because it allows them to find the answer more efficiently. One of them (U9) reported: "I prefer visual explanations because I retrieve faster the answer that I am looking for". Six participants 


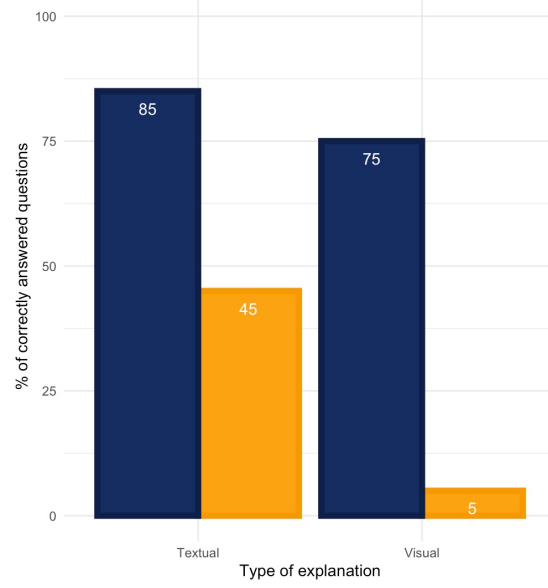

(a) Percentage of correct answers to tasks

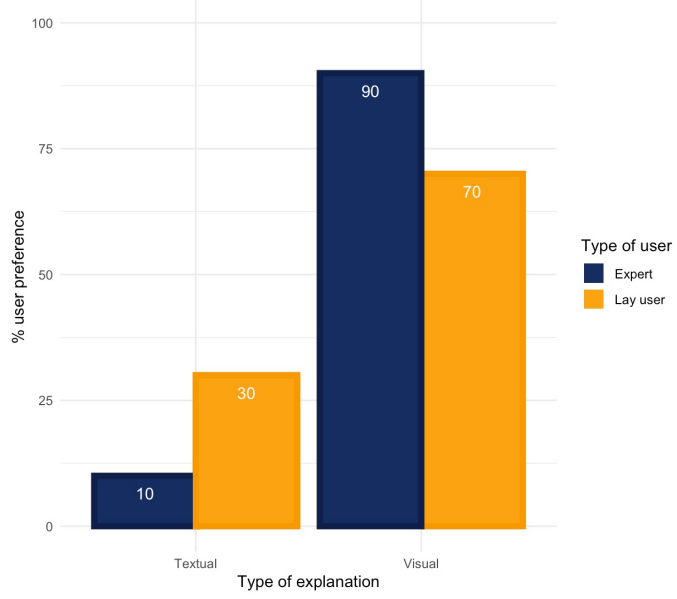

(b) Preference for visualization

Fig. 3. Textual vs. Visual explanations

(4 lay users, 2 experts) preferred visual explanations because "visual explanations are easier to get fast an overview" (U12). Three other lay users also preferred visual explanations, but they gave a suggestion to add additional textual information to the already existing visual explanations. For example, U15 reported: "I prefer visual, but I would prefer to see additional text". However, not everyone preferred the visual explanations. Three participants (1 expert, 2 lay users) preferred textual explanations because they found the visual explanations to be overwhelming.

\section{Q2: IN WHAT USE CASE WOULD YOU USE THE EXPLANATIONS?}

In the answers to this second question, we identified three different themes: Assessing, Learning and the combination of both. A first category of users (3 experts, 6 lay) report that they would use the explanations to assess and improve the current instance they are working on. An example of this is U12 who reports: "I would use it [the explanation] to verify that I am not using too much words or not enough pictures while I am writing”. A second category of users (4 experts, 1 lay user) report that they would use the system to look at predictions of previous instances to learn from the model, and apply this knowledge for future instances. U5 reported this as: "I would look at them after publication so that I know what I could improve for my next article". There is also a third category of six users (3 experts, 3 lay users) which stated they would use the explanations in both scenarios.

\subsection{First User Study Discussion}

The use of the TA methodology allows us to dig deeper into the differences that we noticed in the previous section. One noticeable result is the score of lay users with the visual explanations, where most of the tasks were answered incorrectly. Upon investigating the TA logging, we notice three categories in erroneous reasoning when users gave wrong answers to Task 1 using the visual explanations:

- Wrong interpretation of the graph, where users believe the graph holds different information than it is designed for. U17 stated for example that "high values of a parameter in the graph also mean that the parameter as a whole has a significant impact on reading time". U18 stated: "this graph shows me the value of this article in relation to 
other articles". When looking at the answers in Table 1, we see that users in this category still have a preference for the visual explanations. Due to limited additional information to correct their understanding, these users were unable to correct their understanding, nor correctly answer the task.

- LAY USERS (2): U17, U18 | EXPERT USERS (0): U4

- Giving their answer based on own reasoning, without making explicit use of the visual explanations. All users were asked what their answer was based on, and users in this category explicitly stated that they based their answer solely on personal reasoning.

- LAY USERS (2): U11, U20 | EXPERT USERS (0): -

- Reasoning about an answer, then using incorrect information to confirm this answer. U16 stated: "I believe pictures used would have an impact on reading time. By looking at the explanations, I see it being green so that must mean impactful. Therefore, this and the other green parameter have the highest impact". Two other users, such as U15, reasoned in the same manner but from a different perspective: "I think articles on the first page get read more. If I look at the explanations, I see that it is denoted as red". When asked what the second most impactful parameter was, the user stated: "It must be the other red parameter then". Users inferred a meaning from these colors to fit their predisposed answer, which relates to confirmation bias.

- LAY USERS (5): U12, U14, U15, U16, U19| EXPERT USERS (0): -

When looking at the TA logs during Task 2, we see that even more lay users follow the third category of formulating an answer (U12, U14, U15, U16, U17, U19), with no experts falling under this category during both tasks. Following the definition of Wang et al. [36], this can be described as a flawed backward-oriented reasoning strategy. Using the dichotomous classification for reasoning methods developed by Croskerry [10], the reasoning of lay users during our TA study falls under System I thinking, which is described as fast, low effort and heuristic-based. For inexperienced users however, System I thinking might lead to confirmation bias where a wrong hypothesis gets confirmed by selectively looking for information that supports it $[23,36]$. The TA logs show that lay users made assumptions about the meaning of colors to support and confirm their biased hypothesis (albeit incorrectly). Due to this confirmation bias, lay users formed an answer prematurely without going through other possible parameters, which causes a lack of exploration, and in turn explains why the majority of users answered incorrectly. The lack of exploration was present in 7 lay users (6 using visual, 3 using textual explanations) and 1 expert user (using visual explanations). We can see that this mostly affects lay users, as can be seen in Figure 3a. A reason for this lack of exploration is given by Tsai et al. [35], which state that unfamiliar information might induce a reinforcement effect that causes users to avoid interacting with various content. This explains why the lack of exploration is stronger for lay users in visual explanations as opposed to textual explanations.

When classifying the expert formulation of answering, we see that 7 expert users follow the opposite deductive reasoning approach, where they deduce a possible answer by using explanations, and verify it afterwards through own reasoning (U1, U2, U4, U6, U8, U9, U10). U3 noted for example: "I learned from these graphs that changing the page on which the article is on significantly improves the reading time. When I think about it, this seems logical." This deductive type of reasoning relates to the slower, analytical and high effort System II thinking [10, 36].

Another result we investigated using the TA logs is the user preference for the type of explanation. Figure $3 \mathrm{~b}$ shows that three lay users have a preference for textual explanations. They stated that visual explanations were overwhelming, and felt more confident and comfortable using the textual explanations. We also see that seven lay users still preferred visual over textual explanations. They stated during the TA study that in their opinion, visual explanations helped 
Table 1. First iteration - Categorized answers to the open questions

\begin{tabular}{llll}
\hline Question & Code & Expert & Lay user \\
\hline & Visual - Expertise & U1, U6, U8 & $/$ \\
& Visual - Efficient & U2, U3, U7, U9 & $/$ \\
\multirow{2}{*}{ Q1: Preference } & Visual - Overview & U5, U10 & U12, U17, U18 \\
& Textual - Overwhelming & U4 & U11, U16 \\
& Additional text & $/$ & U15, U19, U20 \\
\hline \multirow{2}{*}{ Q2: Use case } & Assessing instance & U3, U4, U8 & U11, U12, U13, U14, U19, U20 \\
& Learning & U1, U2, U5, U9 & U15 \\
& Both & U6, U7, U10 & U16, U17, U18 \\
\hline
\end{tabular}

them generate a better and deeper understanding of the predictions compared to textual explanations (in other words, they found the system to be more complete). However, they performed poorly on the visual explanations in terms of interpretability.

This illustrates the completeness vs. interpretability tradeoff of explanations that is discussed by Gilpin et al. [12]. Users who felt more comfortable using textual explanations, prefer interpretability over completeness. However, the majority of lay users have a preference for explanations that are more complete, even though it might compromise on interpretability. Out of the seven lay users that preferred visual explanation, three users gave the suggestion to add additional textual explanations to the visual explanations. They stated that it could help users understand the information contained in the visualization and potentially increase interpretability whilst not compromising on completeness. This suggestion will be discussed in-depth in the following section.

\section{SECOND ITERATION: HYBRID EXPLANATIONS}

\subsection{Hybrid explanation design}

From the first iteration we learned that all users preferred the visual explanations except for a few users who found the visual explanations to be too overwhelming (see Section 3.4). Additionally, we learned that especially lay users had difficulties in interpreting the visual explanations (even if they think they do so correctly). To address these issues, we followed the suggestion of three lay users to augment the visual explanations with text to explain the visual elements in text. This new design of explanation will further be denoted as a hybrid explanation. More concretely, the main goal of adding this text is to improve the understanding of the explanation which should result in a higher amount of correctly answered questions than with the visual explanations. We also want to verify whether these textual explanations are able to disprove incorrect interpretations of the visual explanations, as Wang et al. [36] suggest disproving wrong hypotheses as a suitable strategy to overcome confirmation bias. Lastly, we want to verify whether adding textual explanations to a visual explanation does not have a negative effect on experience of the users.

As prior research related to combining both visual and textual explanations is limited [25], we based our design only on previous work done by Hohman et al. [18] who already combined visual explanations with text, but did not provide guidelines on how to do so best. Compared to the design of the visual explanation, we only added two sentences of which the first one explains what the graph represents and the second one states the current value of the parameter and the relation to the other parameters. This addition is indicated with label (4) on Figure 4. 


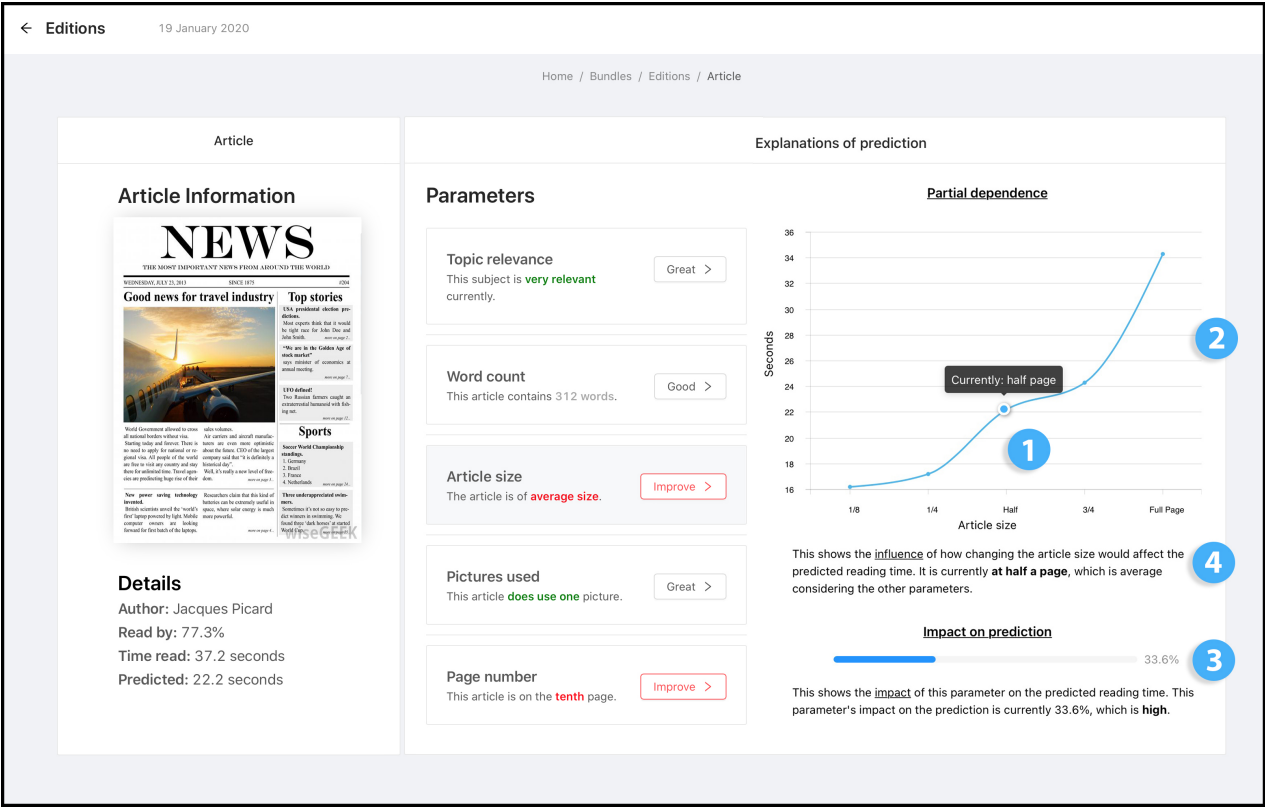

Fig. 4. Hybrid explanations for a selected instance. The current value (1) denotes the C-Local capability, how the selected parameter influences the prediction (2) denotes C-Counter and (3) denotes C-IMPORTANCE. (4) indicates the textual addition in comparison with the visual explanation.

\subsection{Second User Study Methodology}

As indicated before, this user study mainly serves the purpose to assess the performance of the hybrid explanations and to compare this performance to the visual explanations of the previous study. As a consequence, we recruited 20 participants with similar demographics as in the first user study: $10 \mathrm{AI}$ experts (age between 25 and 45, 7M:3F) and 10 lay users (age between 22 and 48, 6F:4M). Additionally, these users had to follow the same methodology as the first user study (Section 3.3) except that they only needed to evaluate one interface and consequently also did not need to answer the open-ended questions at the end of the study. Concretely, this means that they needed to fulfill the same two tasks after which they answered the same post-task questions. Additionally, this user study also used a TA methodology to get insight in the thoughts of the users.

As the previous work about explanations with both text and a PDP is limited [25], we wanted to make sure that our design did not contain usability flaws. To do so, we conducted a pilot user study with 5 usability experts (3M:2F, age between 24 and 35). They followed the same methodology as the main user study described above, but additionally they were asked to answer a SUS-questionnaire [6]. The resulting score of this questionnaire was $\mu_{\text {sus }}=90, \mathrm{SD}_{\text {sus }}=2.23$, by which we can conclude that the system has no major usability flaws [5].

\subsection{Second User Study Results}

As shown in Figure 5, it is clear that the hybrid explanations served its purpose as the performance of lay users improved by $50 \%$ in comparison with the visual explanations. Additionally, it can be seen that the performance of expert users improved by $5 \%$. To verify whether the experience of users changed in the hybrid explanations, we analyzed 


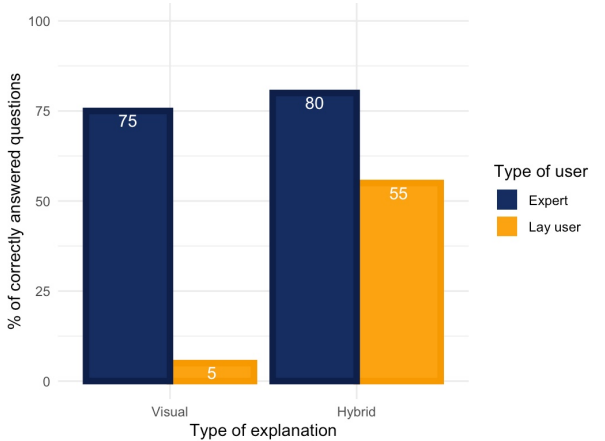

Fig. 5. Hybrid vs. Visual - Percentage of correct answers to tasks

Table 2. Second user study - Significant differences between explanation types to post-task questions

\begin{tabular}{llllll}
\hline & Question & $\mu_{H y b} \pm \sigma_{H y b}$ & $\mu_{V i s} \pm \sigma_{V i s}$ & $\mathrm{U}$ & $\mathrm{p}$ \\
\hline General & Q-EASEOFUsE & $4.13 \pm 0.84$ & $3.63 \pm 0.90$ & 258 & .038 \\
Expert & Q-Time & $1.88 \pm 1.21$ & $3.0 \pm 1.47$ & 27 & .035 \\
Lay & Q-INTUITIVE & $4.13 \pm 0.60$ & $3.38 \pm 1.32$ & 65.5 & .077 \\
\hline
\end{tabular}

Table 3. Second user study - Significant differences between expertise groups to post-task questions

\begin{tabular}{llllll}
\hline & Question & $\mu_{\operatorname{Exp}} \pm \sigma_{\operatorname{Exp}}$ & $\mu_{\text {Lay }} \pm \sigma_{\text {Lay }}$ & $\mathrm{U}$ & $\mathrm{p}$ \\
\hline Expert vs Lay & Q-INTUITIVE & $3.75 \pm 1.07$ & $3.31 \pm 1.17$ & 244.5 & .092 \\
\hline
\end{tabular}

the differences between the post-task questions in the first user study about visual explanations with the post-task questions in this user study about hybrid explanations. We analyzed this data using the Mann-Whitney U test since it does not make an assumption about the underlying distribution of the data, and takes the ordinal nature of the questions into account [26,30]. Similar to the first user study, we used a significance level of $\alpha=0.1$ to detect differences between populations [20]. The results of this analysis revealed four different significant results, all in favor of the hybrid explanation. These results are shown in Table 2.

First, we notice that the perceived ease of use is significantly better for the hybrid explanations. This is revealed both in general as for the lay users, but not for the expert users. Second, expert users perceive that they need less time with the hybrid explanation and that the hybrid explanation is more intuitive than the visual explanation. We can also determine that adding additional textual to visual explanations does not cause an increase in perceived complexity, nor perceived amount of information conveyed by the hybrid explanations.

Additionally, we compared the perception of expert and lay user for both explanations together. As shown in Table 3 this analysis revealed unsurprisingly that expert users perceive explanations as more intuitive to use than lay users. 


\subsection{Second User Study Discussion}

During the TA study, we noticed that neither expert nor lay users expressed a wrong understanding of the figures (as opposed to using visual explanations, Section 3.4), and referred to the textual explanations to either:

- correct their wrong understanding about what information the visual explanations entail. This can be seen by the increase in numbers of correctly answered tasks (Fig. 5). U34 stated: "From the text, I can see that this graph does not show a comparison of how this article relates to other articles as I thought before".

- LAY USERs (4): U34, U35, U36, U39 | EXPERT UsERs (0): -

- learn what information the visual explanations entail in a textual manner. U37 for example stated: "I see a graph, which I don't know what it represents by just looking at it", and after reading the textual explanation stated: "So this learns me that the change in prediction is depicted in the graph".

- LAY Users (4): U31, U33, U37, U38 | ExPert Users (0): -

- confirm their preconceived beliefs about what information the visual explanations hold. These users expressed during the TA study that after reading the textual explanations, they felt more confident using the visual explanations. U22 expressed this as: "This graph shows information $x$, which the textual information confirms".

- LAy users (0): - | Expert Users (4): U21, U22, U25, U27

A distinction can be observed in the use of these added textual explanations according to the user expertise: where experts used the text to confirm their beliefs, lay users mainly used it to learn or correct their understanding of the associated visual explanation. This ties into the disproval of incorrect hypotheses, which as discussed in Section 4.1 should lower the influence of confirmation bias that was previously present with lay users. This is additionally supported by 6 lay users clicking on all parameters when using hybrid explanations, which is an improvement over the previous 3 lay users when using visual explanations. This increase in exploration led to less users giving an answer that was influenced by confirmation bias (only 3 lay users, 0 expert users) and a better understanding of the explanation capabilities (Fig. 5. However, the lack of exploration is still present to some extent with lay users using hybrid explanations, and adding textual explanations alone does not seem to resolve this issue.

\section{DISCUSSION AND CONCLUSION}

In this section, we recap the research questions and discuss the results of the two iterations. Then we provide suggestions for the implementation of explanations based on our observations and discuss limitations and future work opportunities.

\subsection{Answering the research questions}

5.1.1 RQ1: What impact do different types of explanations (textual, visual) have on the interpretation of a predictive algorithm? In iteration 1, we developed visual and textual explanations that present the same explanation capabilities to the user, but in different forms. Results from the first iteration show a strong contrast in the ability to interpret explanations. Users performed more correctly when using textual explanations compared to visual explanations. However, when asked for their preferred explanation type, the majority of participants indicated to prefer visual explanations. Similar to work of Gilpin et al. [12], some users in our first user study indicated to prefer the textual explanations because the visual explanations were perceived as too overwhelming. The main reasons for preferring the visual explanations relate to familiarity and efficiency. Despite this preference, lay users performed much better with textual explanations. 
5.1.2 RQ2: In which way are the differences in interpretation influenced by user expertise? A second aspect of this research pertains to the cross-user differences in perception of the explanations. The most notable difference between expert and lay users is in the Dual Process Model of reasoning, where the majority of lay users followed a faster System I thinking that uses heuristics, and expert users followed a more thorough, analytical System II approach using deductive reasoning.

However, as research by Wang et al. [36] points out, inexperienced users are more susceptible to biases when using System I thinking. This bias presented itself in our research in the form of confirmation bias, where lay users with a preconceived hypothesis tended to favor information that confirms said hypothesis. If the presence of biases is not accounted for, we saw that it limits the capability of lay users correctly using and interpreting the explanations. We also noticed a reinforcing effect when lay users interacted with unfamiliar information (mainly visual explanations), which caused a lack of interaction and exploration and resulted in a poor performance.

5.1.3 RQ3: What might potentially minimize these differences in interpretation? To address differences in interpretation, we designed hybrid explanations that combine the use of textual and visual explanations. Results from the second user study show that lay users performed significantly better when using hybrid explanations compared to visual explanations. As for the experts, no significant change in interpretability was detected ( $80 \%$ of tasks correctly answered compared to $75 \%$ using visual explanations), which seems to suggest that adding textual explanations seems to mostly benefit the interpretability of lay users. However, we do see a benefit for expert users in terms of increase in perceived intuitiveness and a decrease in perceived amount of time needed when compared to visual explanations.

\subsection{Suggestions for the implementation of explanations}

5.2.1 Tailoring explanations to the expertise level of end-users. A first conclusion this research draws is the user expertise being an important moderator in explanation interpretability and use. This confirms that a "one-size-fits-all" method for generating explanations does not exist (in their current state), and user expertise should be taken into account when adapting the type of explanations to the potential end user, as discussed in Section 2. Potential biases that limit the user's interpretability need to be acknowledged and overcome as well. Since these biases may be specific to a certain expertise group, it is important to tailor the solutions that address biases to the expertise group that is affected by them.

5.2.2 Combined use of textual and visual explanations. A second conclusion is that some users potentially prefer a type of explanation that they perform worse with, replicating an earlier identified trade-off between completeness and interpretability [12]. This research identified several additional shortcomings that were mainly associated with lay users (e.g. cognitive bias, information overload) when using these visual explanations. In response, a new method for generating explanations was proposed that improved on some aspects where visual explanations were lacking. This novel approach proved to be a step in the right direction in minimizing the differences in interpretation between different expertise groups. Results show that adding textual explanations to existing visual explanations proves to be beneficial for expert users in terms of perceived intuitiveness and time needed to interpret the explanations compared to purely visual explanations. For lay users, adding textual explanations even proved to be necessary as it helped them to learn or correct their understanding of visual explanations, and reduce the presence of confirmation bias during their reasoning process. 


\subsection{Limitations and Future Work}

A benefit during this research was the use of TA, which gave insight into how different expertise groups used explanations. However, this excludes the use of additional useful metrics along which the explanations can be compared, such as cognitive load or effective time needed. These might potentially show significant differences between explanation types, expertise groups or both. Future work might also investigate what additional measures can be implemented to further mitigate differences between expertise groups (such as addressing confirmation bias specifically with lay users). A final suggestion is to compare more complex visual and textual explanations that include C-NN and C-Comparison capabilities, to see what effect these might have on different expertise groups.

\section{REFERENCES}

[1] Amina Adadi and Mohammed Berrada. 2018. Peeking inside the black-box: A survey on Explainable Artificial Intelligence (XAI). IEEE Access 6 (2018), 52138-52160.

[2] Ahmed Alqaraawi, Martin Schuessler, Philipp Weiß, Enrico Costanza, and Nadia Berthouze. 2020. Evaluating saliency map explanations for convolutional neural networks: a user study. In Proceedings of the 25th International Conference on Intelligent User Interfaces. 275-285.

[3] Alejandro Barredo Arrieta, Natalia Díaz-Rodríguez, Javier Del Ser, Adrien Bennetot, Siham Tabik, Alberto Barbado, Salvador García, Sergio Gil-López, Daniel Molina, Richard Benjamins, et al. 2020. Explainable Artificial Intelligence (XAI): Concepts, taxonomies, opportunities and challenges toward responsible AI. Information Fusion 58 (2020), 82-115.

[4] Alejandro Barredo Arrieta, Natalia Díaz-Rodríguez, Javier Del Ser, Adrien Bennetot, Siham Tabik, Alberto Barbado, Salvador García, Sergio Gil-López, Daniel Molina, Richard Benjamins, Raja Chatila, and Francisco Herrera. 2019. Explainable Artificial Intelligence (XAI): Concepts, Taxonomies, Opportunities and Challenges toward Responsible AI. arXiv:1910.10045 [cs.AI]

[5] Aaron Bangor, Philip T Kortum, and James T Miller. 2008. An empirical evaluation of the system usability scale. Intl. fournal of Human-Computer Interaction 24, 6 (2008), 574-594.

[6] John Brooke. 1996. SUS: a "quick and dirty'usability. Usability evaluation in industry (1996), 189.

[7] Zana Buçinca, Phoebe Lin, Krzysztof Z Gajos, and Elena L Glassman. 2020. Proxy tasks and subjective measures can be misleading in evaluating explainable AI systems. In Proceedings of the 25th International Conference on Intelligent User Interfaces. 454-464.

[8] Margaret Burnett. 2020. Explaining AI: fairly? well?. In Proceedings of the 25th International Conference on Intelligent User Interfaces. 1-2.

[9] Béatrice Cahour and Jean-françois Forzy. 2009. Does projection into use improve trust and exploration ? An example with a cruise control system. Safety Science 47, 9 (2009), 1260-1270. https://doi.org/10.1016/j.ssci.2009.03.015

[10] Pat Croskerry. 2016. A Universal Model of Diagnostic Reasoning. February (2016). https://doi.org/10.1097/ACM.0b013e3181ace703

[11] Fatih Gedikli, Dietmar Jannach, and Mouzhi Ge. 2014. How should I explain? A comparison of different explanation types for recommender systems. International fournal of Human-Computer Studies 72, 4 (2014), 367-382.

[12] Leilani H Gilpin, David Bau, Ben Z Yuan, Ayesha Bajwa, Michael Specter, and Lalana Kagal. 2018. Explaining explanations: An overview of interpretability of machine learning. In 2018 IEEE DSAA. IEEE, 80-89.

[13] Oscar Gomez, Steffen Holter, Jun Yuan, and Enrico Bertini. 2020. ViCE: visual counterfactual explanations for machine learning models. In Proceedings of the 25th International Conference on Intelligent User Interfaces. 531-535.

[14] Peter Goos and Bradley Jones. 2011. Optimal Design of Experiments: A Case-Study Approach. https://doi.org/10.1002/9781119974017

[15] David Gunning. 2017. Explainable artificial intelligence (xai). Defense Advanced Research Projects Agency (DARPA), nd Web 2 (2017), 2.

[16] Jonathan L Herlocker, Joseph A Konstan, and John Riedl. 2000. Explaining collaborative filtering recommendations. Proceedings of CSCW00 pages (2000), 241-250. http://portal.acm.org/citation.cfm?doid=358916.358995

[17] Fred Hohman, Andrew Head, Rich Caruana, Rob DeLine, and Steven Drucker. 2019. Gamut: A Design Probe to Understand How Data Scientists Understand Machine Learning Models. In SIGCHI. ACM.

[18] Frederick Hohman, Arjun Srinivasan, and Steven Drucker. 2019. TeleGam: Combining Visualization and Verbalization for Interpretable Machine Learning. 151-155. https://doi.org/10.1109/VISUAL.2019.8933695

[19] Dong Huk Park, Lisa Anne Hendricks, Zeynep Akata, Anna Rohrbach, Bernt Schiele, Trevor Darrell, and Marcus Rohrbach. 2018. Multimodal explanations: Justifying decisions and pointing to the evidence. In Proceedings of the IEEE Conference on Computer Vision and Pattern Recognition. $8779-8788$.

[20] Jae-Hoon Kim and In Choi. 2019. Choosing the Level of Significance: A Decision-theoretic Approach. Abacus (11 2019). https://doi.org/10.1111/ abac. 12172

[21] Pigi Kouki, James Schaffer, Jay Pujara, John O’Donovan, and Lise Getoor. 2019. Personalized explanations for hybrid recommender systems. In Proceedings of the 24th International Conference on Intelligent User Interfaces. 379-390.

[22] Josua Krause, Adam Perer, and Kenney Ng. 2016. Interacting with Predictions: Visual Inspection of Black-box Machine Learning Models. $5686-5697$. https://doi.org/10.1145/2858036.2858529 
[23] Geoffrey K Lighthall and Cristina Vazquez-guillamet. 2015. Understanding Decision Making in Critical Care. 13, 3 (2015), 156-168. https: //doi.org/10.3121/cmr.2015.1289

[24] Tim Miller. 2019. Explanation in artificial intelligence: Insights from the social sciences. Artificial Intelligence 267 (2019), 1 - 38 . https://doi.org/10. 1016/j.artint.2018.07.007

[25] Sina Mohseni, Niloofar Zarei, and Eric D. Ragan. 2018. A Survey of Evaluation Methods and Measures for Interpretable Machine Learning. CoRR abs/1811.11839 (2018). arXiv:1811.11839 http://arxiv.org/abs/1811.11839

[26] Todd Neideen and Karen Brasel. 2007. Understanding Statistical Tests. Fournal of surgical education 64 (03 2007), 93-6. https://doi.org/10.1016/j. jsurg.2007.02.001

[27] Ingrid Nunes and Dietmar Jannach. 2017. A systematic review and taxonomy of explanations in decision support and recommender systems. User Modeling and User-Adapted Interaction 27, 3-5 (2017), 393-444.

[28] Alice Pavaloiu. 2016. The Impact of Artificial Intelligence on Global Trends. (12 2016)

[29] Mireia Ribera Turró and Agata Lapedriza. 2019. Can we do better explanations? A proposal of User-Centered Explainable AI.

[30] Philip Sedgwick. 2012. Parametric v non-parametric statistical tests. BMF (online) 344 (03 2012), e1753. https://doi.org/10.1136/bmj.e1753

[31] Fabian Sperrle, Mennatallah El-Assady, Grace Guo, Duen Horng Chau, Alex Endert, and Daniel Keim. 2020. Should We Trust (X) AI? Design Dimensions for Structured Experimental Evaluations. arXiv preprint arXiv:2009.06433 (2020).

[32] Aaron Springer and Steve Whittaker. 2019. Making Transparency Clear. In Algorithmic Transparency for Emerging Technologies Workshop. 5.

[33] Nava Tintarev and Judith Masthoff. 2007. A survey of explanations in recommender systems. In 2007 IEEE 23rd international conference on data engineering workshop. IEEE, 801-810.

[34] Erico Tjoa and Cuntai Guan. 2019. A survey on explainable artificial intelligence (xai): Towards medical xai. arXiv preprint arXiv:1907.07374 (2019).

[35] Chun Hua Tsai and Peter Brusilovsky. 2018. Beyond the ranked list: User-driven exploration and diversification of social recommendation. International Conference on Intelligent User Interfaces, Proceedings IUI (2018), 239-250. https://doi.org/10.1145/3172944.3172959

[36] Danding Wang, Qian Yang, Ashraf Abdul, and Brian Y Lim. 2019. Designing theory-driven user-centric explainable AI. In Proceedings of the 2019 CHI conference on human factors in computing systems. 1-15.

[37] RF Woolson. 2007. Wilcoxon signed-rank test. Wiley encyclopedia of clinical trials (2007), 1-3. 